\title{
FLUXOGRAMA MULTIPROFISSIONAL DE ATENDIMENTO EM PUERICULTURA PARA A EQUIPE DE ESTRATÉGIA DE SAÚDE DA FAMÍLIA
}

\author{
Rosane Gomes de Oliveira'; Giselia Aparecida dos Reis Bellinaso²; Maria Isabel \\ Quadros da Silveira Flores ${ }^{3}$; Rejane Bermudes Costa Beber ${ }^{4}$; Andreia Stankiewicz ${ }^{5}$; \\ Dirce Stein Backes ${ }^{6}$
}

\section{RESUMO}

Objetivo: Apresentar e discutir um fluxograma multiprofissional de atendimento em puericultura para a equipe de estratégia de saúde da família, desenvolvido como produto de apresendizagem para a disciplina de Prática Orientda do Mestrado Profisisonal em Saúde Materno Infantil da Universidade Franciscana. Método: Trata-se de um relato de experiência relacionado ao desenvolvimento de um fluxograma multiprofissional de atendimento em puericultura, cuja demanda é originária de discussões interprofissionais e mobilizada a partir da seguinte questão de pesquisa: Como as consultas de puericultura podem ser otimizadas pela equipe de Estratégia de Saúde da Familia? Resultados: Os resultados foram organizados, apresentados e discutidos, a partir de duas unidades temáticas, quais sejam: Da concepção à estruturação do fluxograma de atendimentos em puericultura; Da estruturação à discussão da puericultura como boa prática no cuidado à criança. Conclusão: O fluxograma multiprofissional de atendimento em puericultura, desenvolvido na perspectiva interprofissional e à luz do pensamento da complexidade, busca atentar para a singularidade e a multidimensionalidade do cuidado em saúde à criança e a sua família.

Palavras-chave: Puericultura; Estratégias da Saúde da Família; Desenvolvimento da Criança.

Eixo Temático: Atenção Integral e Promoção à Saúde (AIPS):

\section{INTRODUÇÃO}

O presente trabalho versa sobre como as consultas pediátricas podem ser programadas pela equipe da Estratégia de Saúde da Familia (ESF), sendo possível adaptar

\footnotetext{
${ }^{1}$ Mestranda do curso Profissional Saúde Materno Infantil, pela UFN. E-mail: rosane.oliveira@ufn.edu.br

2 Mestranda do curso Profissional Saúde Materno Infantil, pela UFN. E-mail: giseliareis.ufn@edu.br

${ }^{3}$ Mestranda do curso Profissional Saúde Materno Infantil, pela UFN. E-mail: maria.quadros@ufn.edu.br

${ }^{4}$ Mestranda do curso Profissional Saúde Materno Infantil, pela UFN. E-mail: rejanebermudes@gmail.com

${ }^{5}$ Mestranda do curso Profissional Saúde Materno Infantil, pela UFN. E-mail: andreia@vivavita.com.br

6 Doutora em Enfermagem. Professora do Mestrado Profissional Saúde Materno Infantil da UFN. E-mail: backesdirce@prof.ufn.edu.br
} 
a agenda para o referido momento, adequando o tempo da consulta às suas necessidades inerentes. Esse processo pode ser qualificado por meio um fluxograma de atendimento dinamizado pela equipe de estratégia de saúde da família.

A puericultura é voltada, principalmente, para os aspectos de prevenção e de promoção da saúde e atua no sentido de manter a criança saudável para garantir seu pleno desenvolvimento, de modo que atinja a vida adulta sem influências desfavoráveis e problemas trazidos da infância. Suas ações priorizam a saúde em vez da doença. Seus objetivos básicos contemplam a promoção da saúde infantil, prevenção de doenças e educação da criança e de seus familiares, por meio de orientações antecipatórias aos riscos de agravos à saúde, podendo oferecer medidas preventivas mais eficazes (DEL CIAMPO, 2006).

O modelo tradicional de puericultura fundamentava-se em abordagens autoritárias e em receitas prontas. Atualmente, no entanto, esse processo foi revisitado à luz de novos referenciais que consideram a autonomia e a singularidade de cada criança. Nessa direção, a puericultura não é mais uma prática disciplinar controladora, mas um cuidado multidisciplinar que passou a considerar o caráter multidimensional e multiprofissional do cuidado em saúde (SANTOS; PUCCIN, 2012).

A puericultura tem, portanto, por finalidade oferecer cuidados específicos à criança, desenvolvendo ações de prevenção e promoção de saúde para que cresçam e se tornem adultos saudáveis, representando uma importante ferramenta de acompanhamento e vigilância à saúde da criança (SILVA, SILVA; FIGUEIREDO, 2017). O acompanhamento planejado da criança, em associação às ações de monitoramento das doenças, bem como o estímulo ao aleitamento materno, imunização e orientação alimentar, contribui na promoção, qualidade de vida e crescimento e desenvolvimento saudável da criança (BRANQUINHOS; LANZA, 2018).

Objetiva-se, com base no exposto, apresentar e discutir um fluxograma multiprofissional de atendimento em puericultura para a equipe de estratégia de saúde da família, desenvolvido como produto de apresendizagem para a disciplina de Prática Orientda do Mestrado Profisisonal em Saúde Materno Infantil da Universidade Franciscana. 


\section{METODOLOGIA}

Trata-se de um relato de experiência relacionado ao desenvolvimento de um fluxograma multiprofissional de atendimento em puericultura, cuja demanda é originária de discussões interprofissionais e mobilizada a partir da seguinte questão de pesquisa: Como as consultas de puericultura podem ser otimizadas pela equipe de Estratégia de Saúde da Familia?

O fluxograma foi desenvolvido a partir de uma revisão da literatura, a qual permite a identificação, a sintese e o compilamento de informações acerca de uma determinada temática (GARUZI et al., 2014). A revisão de literatura é uma ferramenta abrangente que permite articular pensamento e construções, como levantar dados, sintese, analise e avaliação dos resultados para melhor evidência ciêntificas (GALVÃO, PEREIRA, 2014).

O processo de aprofundamento teórico, as discussões interprofissionais e o desenvolvimento do fluxograma foi desenvolvido entre os meses de agosto de setembro de 2021, como produto de aprendizagem da disciplina de Prática Orientada do Mestrado Profissional em Saúde Materno Infantil da Universidade Franciscana. Para esta atividade, os mestrandos foram organizados em grupos de trabalho e instigados, à luz do pensamento da complexidade, a discutirem e apresentarem estratégias prospectivas para a melhoria da qualidade do cuidado em saúde.

\section{RESULTADOS E DISCUSSÃO}

Os resultados foram organizados, apresentados e discutidos, a partir de duas unidades temáticas, quais sejam: Da concepção à estruturação do fluxograma de atendimentos em puericultura; Da estruturação à discussão da puericultura como boa prática no cuidado à criança.

\section{Da concepção à estruturação do fluxograma de atendimentos em puericultura}

Quanto maior o conhecimento da equipe e da família, maior a capacidade de contextualização, aceitação de novas práticas e mudanças, para maior satisfação das famílias, equipe motivada e trabalho mais organizado, pois a puericultura é uma prática fundamental para a equipe da enfermagem garantindo a promoção de saúde da criança, não somente no adoecimento, mas também como um porgrana de prevenção. 
A ánalise permitiu criar um modelo rotina que venha contribuir para que a equipe de enfermagem/saúde adquira mais segurança nesta prática. Segue categorias de suporte abaixo juntamente com o fluxograma de organização da equipe.

Reservar dois horários de consultas normais para a primeira consulta da criança garantirá mais tempo do profissional com a família. Até porque os pais apresentam muitas dúvidas, gostam de serem ouvidos e acolhidos nas consultas.

Ao planejar o fluxo de atendimento da puericultura que melhor se adapte ao seu contexto, os profissionais de saúde devem lembrar que atenção á saúde deve ser personalizada. Os procedimentos devem ser adaptados as necessidades da criança e / ou família, dependendo de fatores de risco e resiliência e, evidentemente da estrutura e recursos de saúde, compartilhando as consultas com todos os profissionais da equipe, médico pediatra, enfermagem, fonoaudióloga, fisioterapeuta, nutricionista. Incentivo á comunicação entre os diferentes profissionais, a fim de qualificar a assistência á criança.

É importante avaliar no novo Sistema de Informação em Saúde da Atenção Básica SISAB, o número de crianças, especialmente menores de 5 anos, para planejar o fluxo de atendimento de puericultura e agendar conforme a necessidade da realidade local.

Figura 1 - Fluxograma de atendimentos em puericultura.

\section{FLUXOGRAMA DE ATENDIMENTOS}

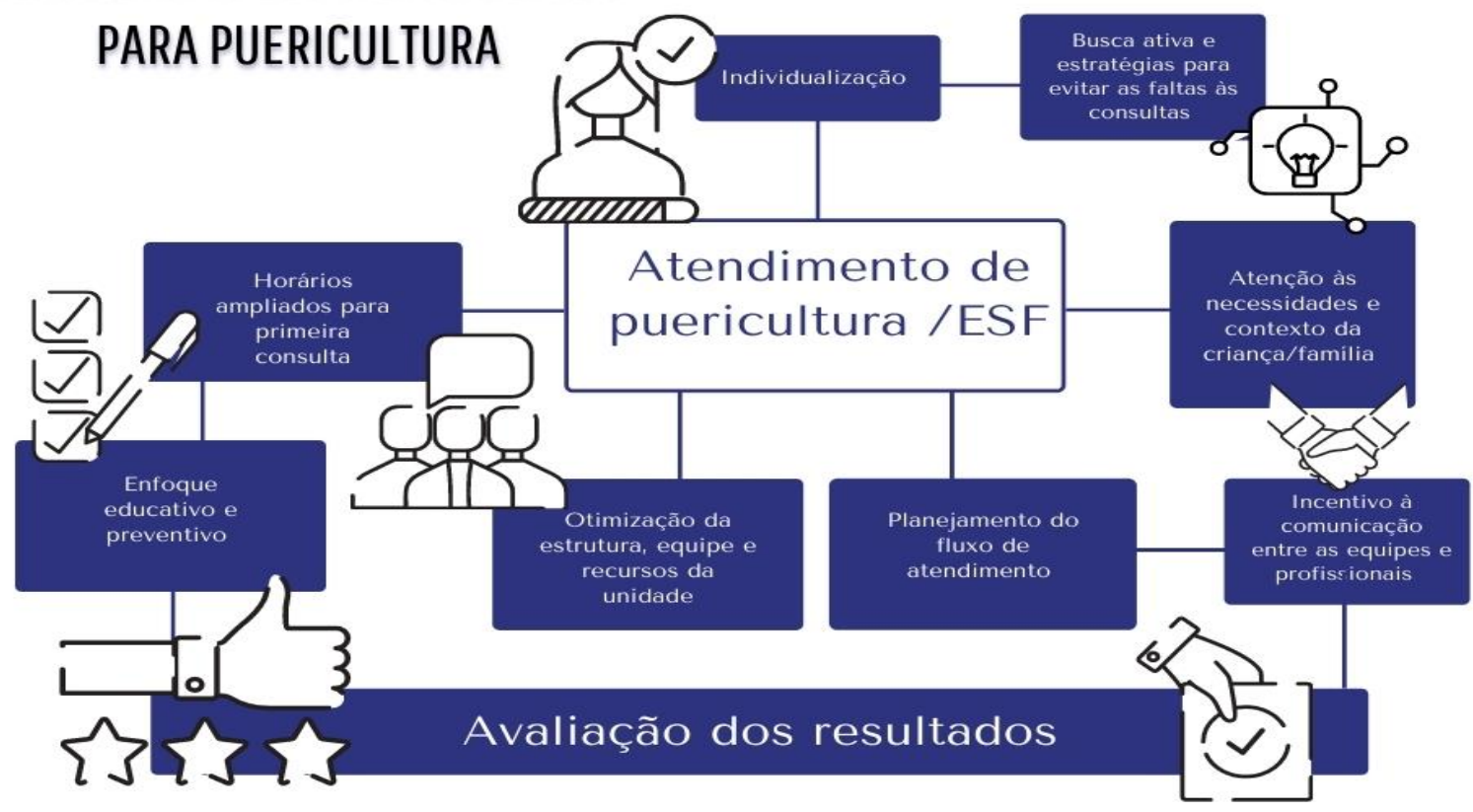

Fonte: elaborado pelas autoras. 


\section{Da estruturação à discussão da puericultura como boa prática no cuidado à criança}

No Brasil, utiliza-se um instrumento para o acompanhamento de saúde da criança, que antes era definido como Cartão da Criança (CC) e tinha como objetivo o acompanhamento individual de crianças nas unidades de saúde para diagnóstico possível de desnutrição energético-proteica utilizando o peso como medida, até os cincos anos de idade (BRASIL, 2002). Tal instrumento passou por algumas modificações sendo hoje utilizada a Caderneta da Criança - Passaporte da Cidadania, um documento que permitirá uma atenção integral à criança não só pelos profissionais de Saúde, mas também pelos da Educação e Assistência Social, no âmbito do SUS (BRASIL, 2019).

Nos serviços de atenção básica, os profissionais que realizam o pré-natal frequentemente são os que seguirão acompanhando a família durante a puericultura da criança. Dessa forma, cria-se um vínculo entre a equipe de saúde e a família do recémnascido para o acompanhamento da criança o qual inicia-se desde o pré-natal (DEMOTT et al., 2006)

O Ministério da Saúde recomenda sete consultas de rotina no primeiro ano de vida (na primeira semana de vida, no $1^{\circ}$ mês, $2^{\circ}$ mês, $4^{\circ}$ mês, $6^{\circ}$ mês, $9^{\circ}$ mês e $12^{\circ}$ mês), além de duas consultas no $2^{\circ}$ ano de vida (no $18^{\circ}$ e no $24^{\circ}$ mês) e, a partir do $2^{\circ}$ ano de vida, consultas anuais, próximas ao mês do aniversário.

Essas faixas etárias são selecionadas porque representam momentos de oferta de imunizações e de orientações de promoção de saúde e prevenção de doenças. As crianças que necessitem de maior atenção devem ser vistas com maior frequência (INSTITUTE, 2012; PORTO ALEGRE, 2004; BRASIL, 2005; ROURKE et al., 2006; BEHRMAN; KLIEGMAN; JENSEN, 2003).

De acordo com a Sociedade Brasileira de Pediatria a primeira consulta do RN deverá ocorrer na sua primeira semana de vida. Este momento torna-se propício para estimular e auxiliar a família nas dificuldades do aleitamento materno exclusivo e verificar a realização da triagem neonatal (teste do pezinho) e para estabelecer ou reforçar a rede de apoio à família. Ainda, a primeira consulta serve para auxiliar a formação ou o fortalecimento do vínculo entre os pais e o bebê, realizar orientações gerais sobre os cuidados com o RN, prevenção de acidentes, orientações para o calendário de imunizações e combinar o calendário de consultas subsequentes (BRASIL, 2004; SBP, 2006; BRASIL, 2012). 
Como a primeira consulta da criança pode ser programada pela equipe, é possível adaptar a agenda para o referido momento, adequando o tempo da consulta às suas necessidades inerentes. Por exemplo: o procedimento de reservar dois horários de consultas normais para a primeira consulta do $\mathrm{RN}$ garantiria mais tempo do profissional com a família (BRASIL, 2012).

A consulta de puericultura é um acompanhamento cuidadoso do crescimento e do desenvolvimento da criança pela equipe de saúde (inclusive com busca de faltosos), com um olhar biopsicossocial não só para a criança, mas também para as condições do contexto de saúde e de vida de sua mãe e família. Além do aconselhamento em relação aos hábitos alimentares, orientações antecipatórias, orientações para o calendário de imunizações, prevenção de lesões não intencionais, prevenção de infecção viral respiratória, aconselhamento para realização de atividade física, prevenção ao uso de drogas e identificação de problemas ou riscos para saúde (BRASIL, 2012).

As famílias compreendem e conhecem a importância do acompanhamento ao crescimento e desenvolvimento infantil. Por outro lado, deparam-se com limitações para aderir à puericultura decorrentes de barreiras institucionais, com relevância ao planejamento das equipes e a (in) flexibilidade para agendamentos das consultas (REZER; SOUZA; FAUSTINO, 2020).

\section{CONCLUSÃO}

O fluxograma multiprofissional de atendimento em puericultura, desenvolvido na perspectiva interprofissional e à luz do pensamento da complexidade, busca atentar para a singularidade e a multidimensionalidade do cuidado em saúde à criança e a sua família.

O acompanhamento sistemático das consultas realizadas na atenção básica é incorporado as políticas públicas e a caderneta da criança torna-se uma ferramenta de registro de indicadores de crescimento e desenvolvimento, possibilitando a comunicação entre os profissionais de saúde de diferentes serviços e a articulação entre os membros da equipe juntamente com a família. Estas estratégias estimulam ações de proteção, promoção, tratamento e recuperação, sendo isto fundamental para que a criança cresça e se desenvolva de forma saudável especiamente nos primeiros anos de vida.

Apesar das famílias entenderem a importância da puericultura, muitas vezes encontram barreiras, tais como a dificuldade de acesso, ou ainda a não flexibilidade de 
EDUCAÇÃO, SAÚDE

ETECNOLLCIA

26 A 28 DE OUTUBRO DE 2021

agendamento das consultas. A puericultura é de suma importância para o acompanhamento da criança e seu desenvolvimento e deve ser incentivada por todos os profissionais da atenção básica.

\section{REFERÊNCIAS}

BEHRMAN, R.E., KLIEGMAN, R.M, JENSEN, H.B. Nelson textbook of pediatrics. 17. ed. Philadelphia: Saunders, 2003.

BRANQUINHOS, I.D., \& LANZA, F. M. (2018). Saúde da criança na atenção primária: evolução das políticas brasileiras e a atuação do enfermeiro. Revista de Enfermagem do Centro-Oeste Mineiro, 8, 2753.

BRASIL. Ministério da Saúde. Cadernos de atenção básica, saúde da criança: acompanhamento do crescimento e desenvolvimento infantil. Brasília, DF, 2002.

BRASIL, Ministério da Saúde. Secretaria de Atenção à Saúde. Departamento de Atenção Básica. Saúde da criança: crescimento e desenvolvimento / Ministério da Saúde. Secretaria de Atenção à Saúde. Departamento de Atenção Básica. - Brasília: Ministério da Saúde, 2012.272 p.: il. - (Cadernos de Atenção Básica, no 33).

BRASIL, Ministério da Saúde. Secretaria de Atenção à Saúde. Departamento de Ações Programáticas Estratégicas. Agenda de compromissos para a saúde integral da criança e redução da mortalidade infantil. Brasília, 2004.

BRASIL. Ministério da Saúde. Caderneta de saúde da criança: Passaporte da cidadania. $12^{\mathrm{a}}$ ed. Brasília, DF, 2019.

DEL CIAMPO, L.A. O Programa de Saúde da família e a puericultura. Rev Ciência \& Saúde Coletiva, 11(3): 739-743, 2006. 
DEMOTT, K. et al. Clinical guidelines and evidence. Review for post natal care: routine post natal care of recently delivered women and their babies. London: National Collaborating Center For Primary Care And Royal College of General Practitioners. 2006.

INSTITUTE FOR CLINICAL SYSTEMS IMPROVEMENT. Health Care Guidelines: preventive services for children and adolescents. 2012

PORTO ALEGRE. Secretaria Municipal de Saúde. Serviço de Saúde Comunitária do GHC. A atenção à saúde da criança de zero a cinco anos de idade. Porto Alegre: SMS, 2004.

REZER. F., SOUZA, T.V., \& FAUSTINO, W.R. (2020). Dificuldades dos responsáveis por crianças na adesão a puericultura. Journal Health NPEPS, 5 (1), 338-350.

SANTOS. R.C.K., RESEGUE R., PUCCINI, R. F. Puericultura e a atenção à saúde da criança: aspectos históricos e desafios. Disponível: http://pepsic.bvsalud.org/pdf/rbcdh/v22n2/pt 06.pdf. Acesso: 12/09/2021.

SILVA. D.M., SILVA, J.G.V., \& FIGUEIREDO, C.A.R. (2017). Assistência de enfermagem em puericultura: um estudo bibliográfico. Revista Saber Científico, 6 (1), 46-60.

SOCIEDADE BRASILEIRA DE PEDIATRIA. Manual prático de atendimento em consultório e ambulatório de pediatria. 2006. 